\title{
Parametric model of human body shape and ligaments for patient-specific epidural simulation
}

\author{
Neil Vaughan ${ }^{\mathrm{a}}$, Venketesh N. Dubey ${ }^{\mathrm{a}}$, Michael Y. K. Wee ${ }^{\mathrm{b}}$, Richard Isaacs ${ }^{\mathrm{b}}$ \\ ${ }^{a}$ Faculty of Science and Technology \\ Bournemouth University \\ Talbot Campus, \\ Fern Barrow, \\ Poole, \\ BH12 5BB \\ United Kingdom \\ ${ }^{b}$ Department of Anaesthesia, \\ Poole Hospital NHS Foundation Trust, \\ Poole, \\ BH15 2JB \\ United Kingdom \\ nvaughan@bmth.ac.uk \\ vdubey@bmth.ac.uk \\ m.wee@virgin.net \\ risaacs@ doctors.org.uk \\ Corresponding Author: \\ Dr. Venketesh Dubey (vdubey@bmth.ac.uk) \\ +44 (0) 1202965986
}

Total words in manuscript: 6825

Total words in introduction: 895

Total words in abstract: 319

Number of figures: 18

Number of tables: 7 


\begin{abstract}
Objective: This work builds upon the concept of matching a person's weight, height and age to their overall body shape to create an adjustable three-dimensional model. A versatile and accurate predictor of body size and shape and ligament thickness is required to improve simulation for medical procedures. A model which is adjustable for any size, shape, body mass, age or height would provide ability to simulate procedures on patients of various body compositions.

Methods: Three methods are provided for estimating body circumferences and ligament thicknesses for each patient. The first method is using empirical relations from body shape and size. The second method is to load a dataset from a magnetic resonance imaging scan (MRI) or ultrasound scan containing accurate ligament measurements. The third method is a developed artificial neural network (ANN) which uses MRI dataset as a training set and improves accuracy using error back-propagation, which learns to increase accuracy as more patient data is added. The ANN is trained and tested with clinical data from 23088 patients.

Results: The ANN can predict subscapular skinfold thickness within $3.54 \mathrm{~mm}$, waist circumference $3.92 \mathrm{~cm}$, thigh circumference $2.00 \mathrm{~cm}$, arm circumference $1.21 \mathrm{~cm}$, calf circumference $1.40 \mathrm{~cm}$, triceps skinfold thickness $3.43 \mathrm{~mm}$. Alternative regression analysis method gave overall slightly less accurate predictions for subscapular skinfold thickness within $3.75 \mathrm{~mm}$, waist circumference $3.84 \mathrm{~cm}$, thigh circumference $2.16 \mathrm{~cm}$, arm circumference $1.34 \mathrm{~cm}$, calf circumference $1.46 \mathrm{~cm}$, triceps skinfold thickness $3.89 \mathrm{~mm}$.

for male or female patients aged 8-85. These calculations are used to display a 3D graphics model of the patient's body shape using OpenGL and adjusted by 3D mesh deformations.

Conclusions: A patient-specific epidural simulator is presented using the developed body shape model, able to simulate needle insertion procedures on a 3D model of any patient size and shape. The developed ANN gave the most accurate results for body shape, size and ligament thickness. The resulting simulator offers the experience of simulating needle insertions accurately whilst allowing for variation in patient body mass, height or age.
\end{abstract}

Keywords Body Shape, Deformation model, Patient-specific, Human Model, Epidural, Simulation.

\title{
1 Introduction
}

The patient's body shape makes a difference to the way medical procedures are performed. Medical simulators have become increasingly popular in recent years. Anatomical 3D models have been incorporated into practice for various procedures such as epidural needle insertion, surgical techniques such as laparoscopy, dentistry, and urethral catheterisation. Medical simulators often contain a manikin on which the procedure is performed which generally represents an average body mass but does not change for patients of different size. The procedures can be performed the same way each 
time which does not encapsulate procedural variation due to varying body mass of patients.

In reality, there is a great deal of variations between different patients' sizes and body shapes; a point that has become ever so more prominent due to the recent obesity epidemic. These patient variations greatly affect many anaesthetic and surgical procedures, such as epidural needle insertion for which a longer Tuohy needle may be required for the morbidly obese to traverse additional adipose tissue. Anaesthetists find that successful insertion of an epidural catheter is much harder in overweight and obese parturients due to difficulties in locating the midline of the spine during palpation [1-3]. The Mediseus epidural simulator attempted to encapsulate patient variety with two options for obese or normal size, but recent studies suggest that is not enough to represent the continuous nature of patient variation [4]. Modelling for a patient's body mass index (BMI) is important since obesity is rising to an epidemic level and the Health Survey for England (HSE) showed that in England 26.1\% of all adults were obese in 2010. To address these issues, an adaptable human body shape model is required. A model which is adjustable for any size, shape, body mass, age or height would provide ability to simulate procedures on patients of various body compositions.

Previous related studies include an isomorphic polygon model for describing human body shape [5]. A relationship between BMI and waist-to-hip ratio (WHR) was proposed [6]. Research into modelled clothing has stretched garments to fit onto 3D human models of various body shapes using skeleton-driven volumetric deformation [7]. Moreover, body water volume (BWV) has been estimated from anthropometric measurements [8-10]. Measurements of the human body are often taken by clinicians to provide information on body composition by analysing various metrics. These include circumferences of waist, thighs, hips, BMI derived from body mass and height, WHR or BWV [11]. Body shape descriptors such as apple, pear, hourglass and banana are often used to describe the visual shape caused by varying proportions of the patient's musculoskeletal configuration. All of these are useful to determine various aspects of body composition. Also gender should be taken as an input to a body shape model because males and females with equal weight and height may have different average body circumferences. This work aims to implement such a body shape model.

A patient-specific epidural simulator has been created based on measurements and developed empirical relations using the body shape model, ligament thickness model and graphic visualisation. This provides a patient-specific epidural simulator, able to reconstruct any body shape from patient measurements to provide a more complete scenario in which to learn and practice the epidural procedure. All information describing the shape of the patient's body and ligaments is visualized in 3D using OpenGL with a 
3D mesh. Deformations are applied to the body shape mesh changing the shape to match any particular patient. The increased variation of patient-specific simulation provides a learning tool that offers a better training experience for anaesthetists. This can increase skill levels, improve progression along the learning curve, reduce the number of failed insertions and reduce the risk of harm to patients.

The presented model takes standard anthropometric patient data of body mass, height and age as inputs. Empirical formulae are then developed to calculate body circumferences, total body water (TBW), and WHR for the patient's body. A novel anatomical model of spinal ligaments in the back is then developed for epidural training. Whilst detailed models for other body parts such as pelvis are available [12], no quantitative anatomical model of the ligaments in the back exists in the literature. The ligament model can adjust in size and thickness to match any patient. This includes all layers that the epidural needle passes through during insertion such as skin, subcutaneous fat, supraspinous ligament, interspinous ligament, ligamentum flavum before finally reaching the epidural space. Layer thickness and density are important for epidural simulation because in vivo, each tissue layer causes a different level of force on the needle which is sensed by the operator. This haptic feedback helps guide the epiduralist to 'feel' the ligaments to successfully carry out the procedure.

Two main problems are tackled in this paper: (1) To generate 3D visualisation of body shape to match measurements of a patient's body mass, height, body shape and age. (2) To accurately calculate thickness of modelled spinal ligaments, bone, fat and skin for patients of any size and shape. Novel aspects of this work include the 3D visualisation of body shape adjustable to match any patient size and shape based on clinical metrics, the anatomical model of ligaments of the back for epidural insertion which adjust in size and shape, and the collection of clinical body shape metrics into one system. These are combined into the first patient-specific epidural insertion simulator for any body shape and size.

\section{Methods}

For each patient a set of standard anthropometric measurements are taken. The measurements are inputs to the mathematical model: $m$ (body mass, $\mathrm{kg}$ ), $h$ (height, $\mathrm{cm}$ ), including $a$ (age, years) and $s$ (sex, male/female). Also, a qualitative description of body shape (Apple, Pear, Hourglass or Banana) and number of weeks pregnant are taken if applicable. These are chosen because they are easily achievable yet descriptive anthropometric data. 
Formulae are then applied to calculate the BMI, TBW, fat mass, fat-free mass and to estimate the circumferences of waist, hip, thigh, calf and arm. Thicknesses of ligaments in the back are also calculated including all layers that the epidural needle passes through. These are thickness ( $\mathrm{mm}$ ) of skin, subcutaneous fat, supraspinous ligament, interspinous ligament, ligamentum flavum and epidural space.

Finally a graphical visualisation is displayed using the body shape estimations to generate a visual model matching the shape and size of the patient's body.

\subsection{Body shape empirical relation formulae}

BMI is a standard parameter and is calculated using the existing Eq. 1. It is notable that height has more effect on BMI than body mass. Halving body mass halves BMI whereas halving height quadruples BMI. The same BMI cut-offs are used for both men and women who have different body compositions and due to this BMI underestimates adiposity in women [13].

$B M I=\frac{m}{h^{2}}$

where $B M I=$ Body Mass Index, $m=$ body mass $(\mathrm{kg}), h=$ height $(\mathrm{m})$.

TBW is predicted by Eq. 2 based on the female Watson formula [10]. Alternative methods do exist but are less accurate [14] such as taking 58\% of the body mass, the Hume-Weyers formula [8] or the Chertow formula [15]. TBW increases linearly with height and body mass but in males decreases with age. Low TBW indicates dehydration which dries the skin and causes organs to shrink which could affect needle insertion.

$T B W=-2.097+(0.1069 h)+(0.2466 m)$

where $T B W=$ total body water.

Fat mass $(F M)$ is calculated directly from BMI by Eq. 3 which is based on [14]. Fat-free mass $(F F M)$ is the remainder of body mass when fat mass is subtracted, given in Eq. 4 . Fat mass as percentage of body mass is given by $\left(F M^{*} 100 / \mathrm{m}\right)$. Women have larger quantities of subcutaneous fat deposits than men, and women carry subcutaneous fat in their gluteal region; men carry most of their fat in the abdominal region. In obesity, there is a higher level of ectopic fat accumulating within cells of non-adipose tissue.

$F M=(1.9337 B M I)-26.422$

$F F M=m-F M$

where $F M=$ fat mass $(\mathrm{kg}), F F M=$ fat free mass $(\mathrm{kg})$ 
Body Cell Mass (BCM) is given by Eq. 5, calculated from the fat-free mass [14]. BCM can be given as a percentage of body mass, by $(B C M / m * 100)$.

$B C M=(0.3655 F F M)+4.865$

where $B C M=$ body cell mass $(\mathrm{kg})$

Waist and hip circumferences grow at different rates because BMI is positively correlated with WHR [6]. An additive model of fat deposition is used where fat is deposited at a constant rate on fixed musculoskeletal frames whose waist-to-hip proportions vary from one individual to the next. Therefore as BMI increases, the difference between waist and hip size decreases making the body less curvaceous and the WHR increases, by Eq. 6 .

$W H R=\frac{(2.058 \times B M I+29.670)}{(1.842 \times B M I+56.004)}$

Body descriptors (Apple, Pear, Hourglass, Banana) can be simulated by adjusting the body circumferences to effectively shift mass between body parts. This is calculated by moving proportions of total area from one area to another. An Apple patient has greater proportion of area on hip and waist, less on arm and calf, whereas thighs will remain unaffected. Hourglass will have thinner hip, waist and thighs but larger arms and calves. The total area always remains as $100 \%$ so the modelled body mass is unaffected by changing body descriptor.

Body Adiposity Index (BAI) is used to estimate the amount of body fat without referring to body mass measurement, by a standard formula shown in Eq. 7. The model already calculates fat mass in Eq. 3 but BAI gives a comparable measure taking into account $\mathrm{HC}$.

$B A I=\frac{H C}{h^{1.5}}-18$

where $B A I=$ Body Adiposity Index, $H C=$ Hip circumference $(\mathrm{cm})$

Five body circumferences for each patient are calculated by comparing patient's body mass to the average body mass for their age. The body circumferences of the model are then updated to match the patient by adjusting body circumferences from an average woman, data shown in Table 1, from the study [16]. Adipose tissue in overweight women collects in the gluteal region [13]. This model adds $75.83 \%$ of additional body mass to the waist and hips combined because they have the largest proportion of total area, as in Table 1. 


\begin{tabular}{|l|l|l|l|l|}
\hline Limb & $\begin{array}{l}\text { Range } \\
(\mathrm{cm})\end{array}$ & $\begin{array}{l}\text { Circumfer } \\
\text { ence }(\mathrm{cm})\end{array}$ & $\begin{array}{l}\text { Area } \\
\left(\mathrm{cm}^{2}\right)\end{array}$ & $\begin{array}{l}(\%) \text { of } \\
\text { total area }\end{array}$ \\
\hline Arm & $20-45$ & 32.5 & 84.05 & 4.65 \\
Calf & $30-45$ & 37.5 & 111.90 & 6.19 \\
Thigh & $40-80$ & 55 & 240.72 & 13.31 \\
Waist & $60-120$ & 85 & 574.94 & 31.81 \\
Hip & $80-130$ & 100 & 795.77 & 44.02 \\
\hline
\end{tabular}

Table 1. Body circumferences for an average woman [16].

A method has been developed to calculate thigh, calf, waist and arm circumferences from body mass. Circumferences are treated as circles so their area can be calulated as in Figure 1. The areas are assumed proportional to total body volume, which is proportional to body mass. Therefore the cross-section arm area for a patient of any body mass is calculated by Eq. 8. Finally the geometric relationship between the area and circumference of a circle can be used to calculate arm circumference by Eq. 9. The equation takes body mass into account through the variable $m$ and also age through $\mathrm{am}$.

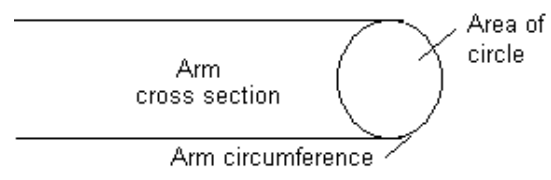

Figure 1. Relationship of arm circumference to body mass.

$A=84.05\left(\frac{m}{a m}\right)$

where $A=$ arm area, $a m=$ average body mass for age.

$C=2 \sqrt{A \pi}$

where $C=$ circumference.

An equation was developed to calculate body mass gain during pregnancy. The effects of pregnancy on body shape are important for epidural simulation. The equation was developed as a best fit to match known weight gain information from [17]. Body mass gain usually starts around 6 weeks post conception (Figure 2). Body mass increase during the following 34 weeks resembles a sigmoid curve [17]. Total net gain by gestational week 40 is $12-15 \mathrm{~kg}$ [18]. By 6 weeks postpartum half of the net gestational gain is lost. By 6-18 months postpartum, body mass is $1-2 \mathrm{~kg}$ heavier than pregravid body mass, 
although $20 \%$ of women retain more than $5 \mathrm{~kg}$ above preconception levels and this occurs more frequently in overweight and obese BMI groups. Taking these facts into account, a net gestational body mass gain $(\mathrm{kg})$ during pregnancy above pregravid body mass is implemented in the model as represented by Eq. 10, the curve of which is visualized in Figure 2. (Note that the net weight does not include the child's weight).

$$
M G=14 \times\left(\frac{1}{1+1.025^{(140-d c)}}\right)-12 \times \sin \left(\frac{90 \times d b}{180}\right)
$$

where $M G$ = body mass gain during pregnancy $(\mathrm{kg}), d c=$ days since conception (0-280), $d b=$ days since birth $(0-180), \sin =$ sine function.

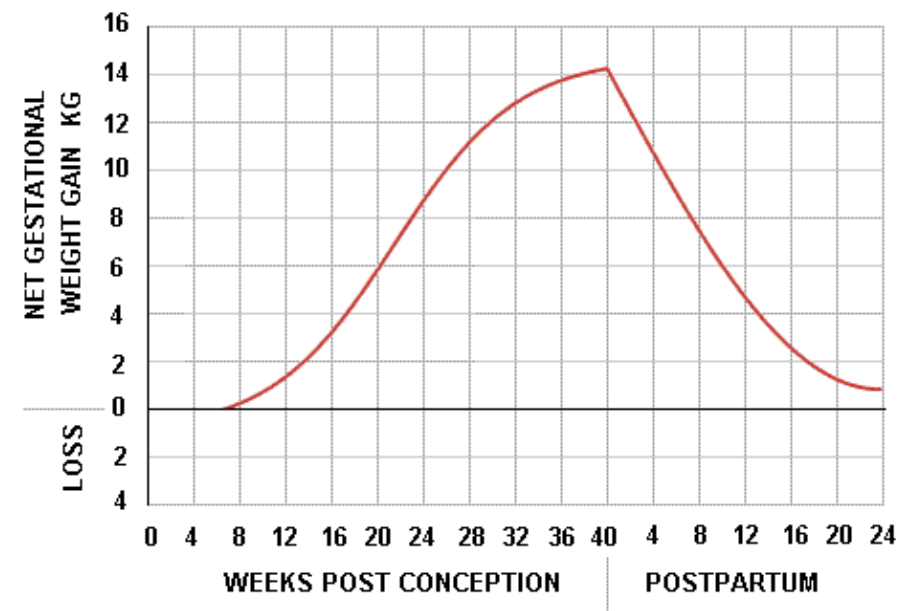

Figure 2. Body mass gain estimation during and after pregnancy.

\subsection{Ligaments thickness estimation}

The thickness of the 5 tissue layers in the back which are penetrated by an epidural needle varies between patients. For accurate simulation of the procedure it is required to estimate the thicknesses for any patient. Studies have provided average thicknesses of the layers in human patients and these data are combined in Table 2, further details are given in our previous work [19]. Measurements of human LF thickness from 10 human cadavers were 3-5mm thick [20]. Also LF was measured by CT images as 2.7-2.9mm at L3-L4 and 3.43.2mm at L5-S1 [21]. MRI studies found LF thickness to be 3-3.5mm in age group 20-29 [22]. The epidural space is a potential space containing blood vessels, lymphatics and nerves, and when the needle enters and injects saline it may open to around $6 \mathrm{~mm}$ before the needle punctures into the dura. Subscapular skinfold thickness calliper gives a reliable indication of body fat [23]. Skin thickness can be estimated with a margin of error between $3 \%$ and $11 \%$ [24]. 


\begin{tabular}{|l|l|l|}
\hline Tissue Layer & $\begin{array}{l}\text { Thickness } \\
(\mathrm{mm})\end{array}$ & $\begin{array}{l}\text { Depth } \\
(\mathrm{mm})\end{array}$ \\
\hline Skin & 3 & 0 \\
Subcutaneous fat & 6 & 3 \\
Supraspinous ligament & 4 & 9 \\
Interspinous ligament & 26 & 13 \\
Ligamentum flavum & 3 & 39 \\
Epidural space & 6 & 42 \\
Dura & 15 & 48 \\
\hline
\end{tabular}

Table 2. Tissue layers thickness and depth approximation based on porcine [19] and human [2022] measurements.

Two skinfold equations are proposed (Eq. 11 and 12) working on the basis that tissue layer thickness is proportional to the radius of the waist. The equations assume that thickness increases proportionally to waist to the third power of radius, because fat collects in the subcutaneous fat layer so thickness increases faster than body mass increase. The value 574.94 used in Equations 11 and 12 is the average waist cross sectional area $\left(\mathrm{cm}^{2}\right)$ from Table 1.

$S S=\left(\sqrt{\frac{574.94 *\left(\frac{m}{a m}\right)}{\pi}} / a r\right)^{3} * a S$

where $S S=$ subscapular skinfold thickness $(\mathrm{mm})$, $a r=$ radius of the average waist circumference, (calculated as $13.52809 \mathrm{~cm}$ ), as = mean subscapular skinfold thickness (calculated as $16.73 \mathrm{~mm}$ ).

$T S=\left(\sqrt{\frac{574.94 *\left(\frac{m}{a m}\right)}{\pi}} / a r\right)^{3} * a t$

where $T S=$ triceps skinfold thickness $(\mathrm{mm}), a t=$ mean subscapular skinfold thickness (calculated as $16.52 \mathrm{~mm}$ ).

An equation to calculate subcutaneous fat is proposed based on Table 2 (Eq. 13). Subcutaneous fat thickness varies between regions of the body. Eq. 13 predicts subcutaneous fat from body fat percentage which is known from Eq. 3. Subcutaneous 
adipose tissue can be reliably measured by CT scan at the L2-L3 and L4-L5 disc spaces [25].

$S F=\frac{\left(\frac{F M}{m}\right) \times 100-18}{3}$

where $S F=$ subcutaneous fat thickness (mm).

Epidural space is assumed to remain the same for patients of various size. The depth of each ligament or layer is determined by the sum of all previous layer thicknesses. The equations give ligament thickness estimations for any patient, but in order to improve the accuracy further, MRI was used to collect data from patients so specific measurements could be made for each patient

\subsection{MRI dataset for ligament measurements}

A function to load MRI ligament thickness measurements from a saved MRI dataset derived from an ongoing clinical trial has been implemented in the simulator software. MRI scans of the lumbar region provide clear images showing each of the ligaments in the back allowing measurement of all ligament thicknesses with high accuracy. Thicknesses are measured in the MRI imaging software by overlaying lines of known length onto the MRI image. These measurements can then be saved into a dataset shown in Table 3. The simulator software can load the MRI dataset and update each ligament thickness with the measurements from MRI. This method allows the simulator to be specific for the actual patient with high accuracy. Once the simulator has loaded the MRI dataset, ligaments are updated and epidural simulation can proceed using the ligaments of thickness matching the measured data.

\begin{tabular}{|l||l||l|}
\hline Known parameters & Calculations & MRI Measurements \\
\hline Body mass & Body mass index (BMI) & Skin thickness \\
\hline Height & Total body water (TBW) & Subcutaneous fat thickness \\
\hline Age & Fat mass (FM) & Supraspinous ligament thickness \\
\hline Gender & Fat-free mass (FFM) & Interspinous ligament thickness \\
\hline \multirow{2}{*}{} & Call mass & Ligamentum flavum thickness \\
\cline { 2 - 2 } & Waist circumference & Epidural space thickness \\
\cline { 2 - 2 } & Hip circumference & \\
\cline { 2 - 2 } & Arm circumference & \\
\cline { 2 - 3 } & Calf circumference & \\
\cline { 2 - 3 } & Thigh circumference & \\
\cline { 2 - 3 } &
\end{tabular}


Table 3. MRI Dataset stored for each patient's parameters.

The MRI dataset allows accurate measurements of ligament thickness to be made for a patient. The lumbar MRI images were obtained for twenty obstetric parturients within twenty four hours after their epidural procedure. MRI was taken by experienced radiologists at Poole Hospital NHS Foundation Trust. The MRI study was approved by National Research Ethics Service (NRES). The MRI images allowed direct measurement of the ligament thicknesses for each patient; however, taking MRI images for each epidural patient would not be viable in the long term due to the expense of MRI procedure. Artificial Neural Networks (ANNs) may offer estimates for patients when MRIs are not available, by basing estimates on known data from learnt patient measurements. The prediction capability could use known data from previous MRIs but could also learn and improve accuracy as new data sets are added over time.

\subsection{ANN for body shape prediction}

An ANN has been implemented aiming to predict the patient's body circumferences and ligament thickness from patient measurements to a greater level of accuracy. Training was completed using clinical data from 23088 patients obtained from NHANES [26]. The data population contains males and females aged 8 to 85 , of all body sizes, with a mixture of ethnicities representative of US national sample taken across 15 counties. Selfidentified ethnicity rates include Mexican American 25\%, Other Hispanic 3\%, NonHispanic White 40\%, Non-Hispanic Black 27\%, Other Race or Multi-Racial 5\%.

The motivation to use ANN was to gain benefit from learning methods which would improve accuracy over time. In the clinical trial each time we obtained a new patient data, this is added into the data set so that the accuracy will improve over time. Also for regression analysis, this can be run each time a new patient data is added to benefit machine learning based on measured data.

A fully connected feed-forward ANN is used, containing no loops, four inputs, eight hidden neurons in one hidden layer and one output. The output which is a single floating point value from 0 to 1 . The learning mechanism is back-propagation of error. There are 4 inputs, body mass, height, age, gender; waist circumference was used as an optional $5^{\text {th }}$ input. Each input consists of up to 100 binary neurons of 1 or 0 to represent the integer value inputs. Each input value including height, age and body mass is converted from a real integer value into binary before being assigned to each of the input neurons, which are either 0 or 1 . The network can generate estimates for waist, arm, calf and thigh circumferences and thickness of skin, fat, supraspinous, interspinous, ligamentum flavum and epidural space. Each output is generated with a separate instance of the ANN. 
The training process was tested by learning the saddle shape equation $\mathrm{z}=\mathrm{x}^{2}-\mathrm{y}^{2}$ shown in Figure 3. This equation was chosen for testing because the hyperbolic paraboloid contains nonlinear curvature in simultaneous dimensions. During training the weights of links between neurons adapt to the calculated error in the presence of input patterns, applied backward from the network output layer through to the input layer. The test resulted into a trained network with output closely resembling the saddle shape (Figure 3) and the achieved performance was within $0.51 \%$ mean error.

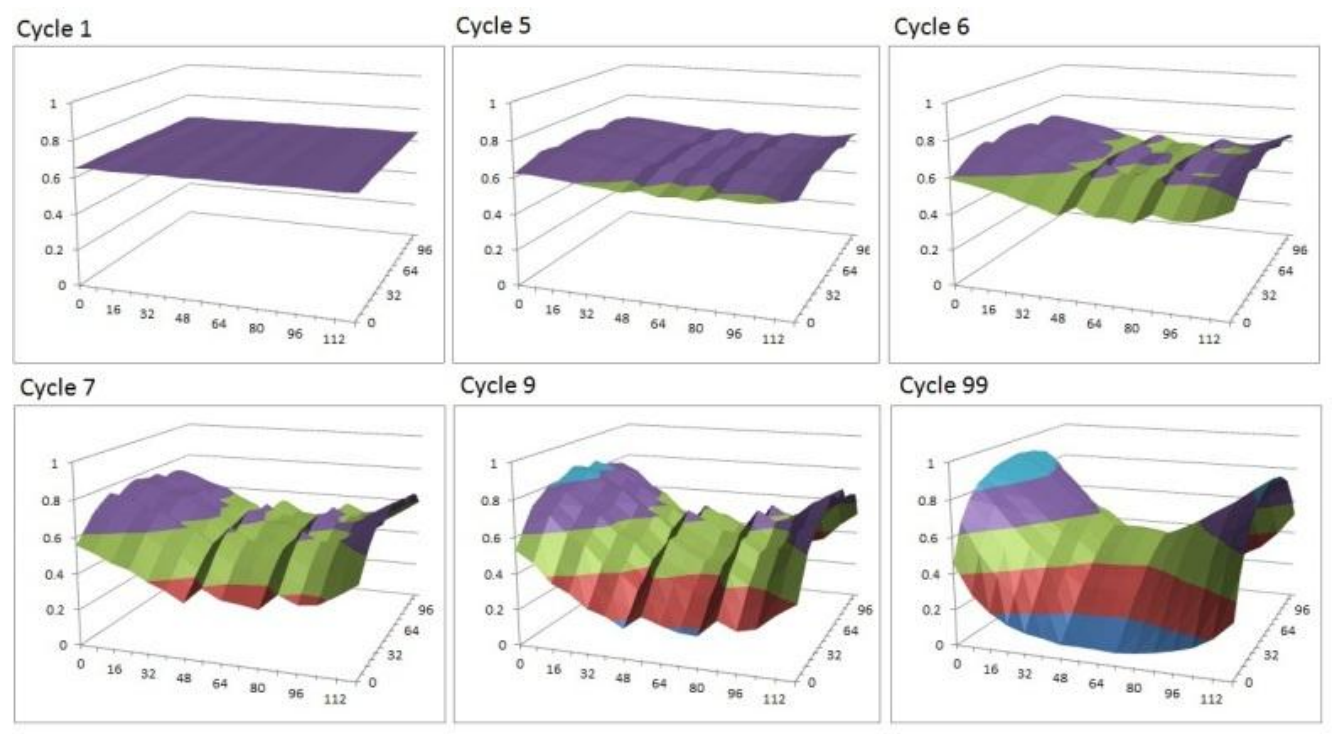

Figure 3. ANN learning equation $\mathrm{z}=\mathrm{x}^{2}-\mathrm{y}^{2}$.

The ANN was then trained and tested using clinical data from 23088 patients. We combined four bi-annual databases from NHANES [26] to create a larger dataset spanning eight years 1999-2006. The data contains a wide range of body shapes, waist circumference varies from $32-175 \mathrm{~cm}$, ages from 8-85. Pregnant patients were excluded from this because due to their changing body shapes, the ANN would need to be trained separately. Patients with incomplete data were excluded. This data was ideal for learning body shape because it includes circumferences of waist, thigh, arm and calf plus two skinfold thicknesses from triceps and subscapular which provides measurement of tissue layer thickness.

The data was collected by trained clinicians following a set protocol to ensure body circumferences were measured the same way with each patient. Arm circumference is measured with tape measure lightly around the arm not compressing the skin, with muscle un-flexed, on the widest part of bicep, perpendicular to arm's long axis. More details are in [26]. 


\subsection{Regression analysis for predicting body circumferences}

As an alternative comparison to ANN, a regression analysis was completed to predict the body circumferences. The regression was done separately for each of the body circumferences; arm, thigh, calf, waist, subscapular and triceps skinfold thicknesses.

The same polynomials were used for regression analysis as were used with the ANN inputs: age, gender, body mass and height. During all analyses the p-values for each independent variable (age, gender, mass, height) was very small (0.003) which confirms there is a relationship between all variables and the outputs, so the null hypothesis (that there is no correlation) can be rejected and this shows that these variables do impact quality. The R-square values were all $>0.8$ and $<0.9$ except for skin thicknesses which were 0.6 , and these R-square values show the regression found a good fit to the data. The Normal Probability plots confirmed that the data set is approximately normally distributed.

Regression analysis identified the intercepts and coefficients for each predicted circumference as shown in Table 4. These values were used to generate the predictions. For example, calf circumference was predicted by regression analysis using the form shown in equation 14 .

$$
Y=21.02-0.027 a+1.187849 g+0.229 m-0.006 h
$$

where $a$ is age, $g$ is gender, $m$ is body mass $(\mathrm{Kg}), h$ is height $(\mathrm{cm})$.

\begin{tabular}{lcccccr}
\hline & Calf & \multicolumn{1}{c}{ Arm } & \multicolumn{1}{l}{ Waist } & \multicolumn{1}{c}{ Thigh } & \multicolumn{1}{c}{ Subscap } & \multicolumn{1}{c}{ Triceps } \\
\hline Intercept & 21.02599 & 24.23286 & 89.1709 & 36.25606 & 31.7848 & 31.81561 \\
Age (years) & -0.02784 & 0.004286 & 0.160655 & -0.08985 & 0.021936 & -0.0271 \\
Gender & 1.187849 & -0.07414 & -0.37736 & 1.997937 & 3.175824 & 7.647241 \\
Mass (Kg) & 0.229066 & 0.277902 & 0.831422 & 0.400678 & 0.427857 & 0.36313 \\
Height (cm) & -0.00695 & -0.08193 & -0.3911 & -0.08038 & -0.29912 & -0.30426 \\
\hline
\end{tabular}

Table 4. Intercepts and coefficients for polynomials from regression analysis.

\section{Results}

The clinical data from NHANES was used to train the ANN -the demographics section gave information on patient gender and age and body examination measurements section gave information on body circumference, weight and height [26]. Data was divided into a training set of 11000 patients and an unseen test data set of 12088 patients. These numbers were chosen to keep the training set big enough to spot patterns in the data 
whilst providing comprehensive accuracy assessment. Generally test sets of $10 \%$ are enough to indicate accuracy. Initially a subset $10 \%$ of the data was used for k-fold crossvalidation to estimate the performance of the predictive model on the independent dataset. K-fold validation gives the advantage that all observations are used for both training and validation, and each observation is used for validation exactly once. ANN inputs were age, body mass, height and gender. Twenty five training cycles were completed with the 11000 patient training set. After each training cycle neuron outputs advanced closer to the measured clinical data. Figure 4 shows the ANN before, during and after training with clinically measured data.
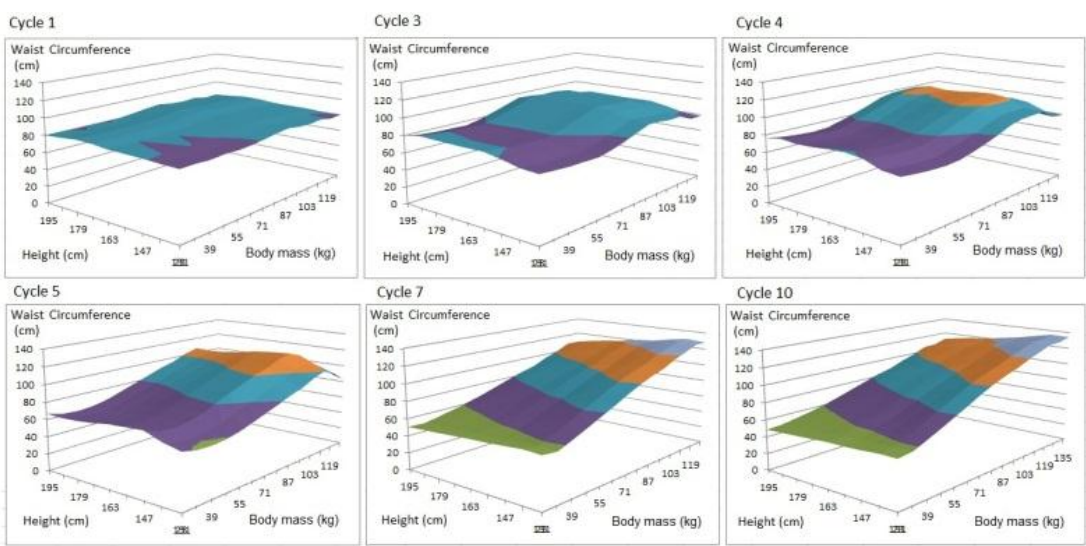

Figure 4. ANN training with clinical data from 23088 patients learning to predict waist circumference.

The accuracy of waist circumference predictions by the ANN grew successively more accurate on each training cycle as shown in the learning curve (Figure 5).

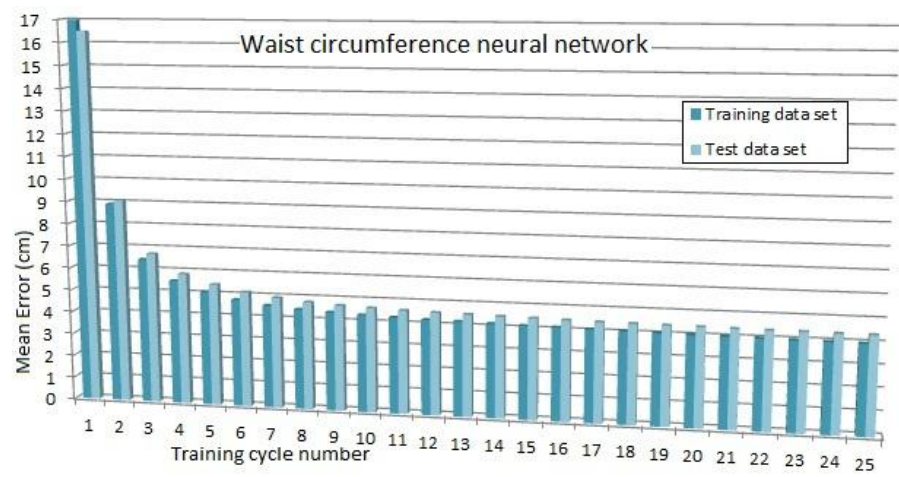

Figure 5. ANN learning curve for waist circumference using clinical data.

Subsequently, the ANN was trained to predict thigh, calf and arm circumference, and skinfold thickness. For each, a graph was generated to visualize output predictions for the entire range of patient body masses and heights (Figure 6). This demonstrates the effect 
of body mass and height on calf circumference and shows that the ANN has learnt the relationship. It shows that body mass is positively correlated with calf circumference. Subscapular skin thickness is negatively correlated with height (Figure 7) and thigh circumference positively correlated with body mass (Figure 8 ).

The ANN has been extended to predict ligament thicknesses using the data from MRI. The MRI scans of the lumber region show accurate thicknesses of all ligaments in the back. Each time a new MRI image is taken, it is added to the training set. The ANN trains on the MRI dataset, to learn the relationship between ligament thickness and patient measurements.

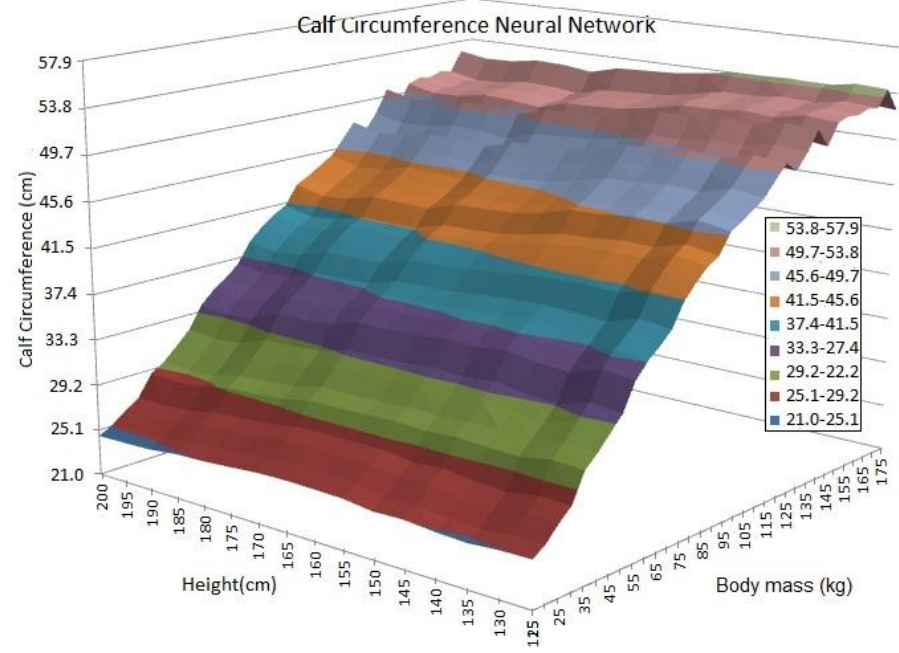

Figure 6. ANN prediction of calf circumference accurate to $1.40 \mathrm{~cm}$. Trained with 23088 patients clinical data.

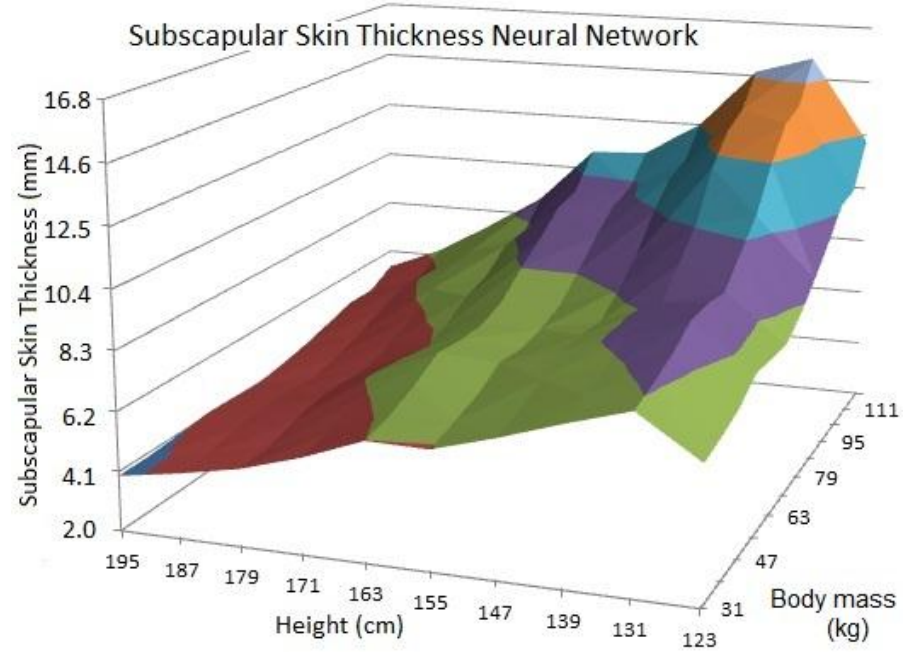

Figure 7. ANN predictions for subscapular skinfold thickness with mean accuracy within $3.54 \mathrm{~mm}$. 


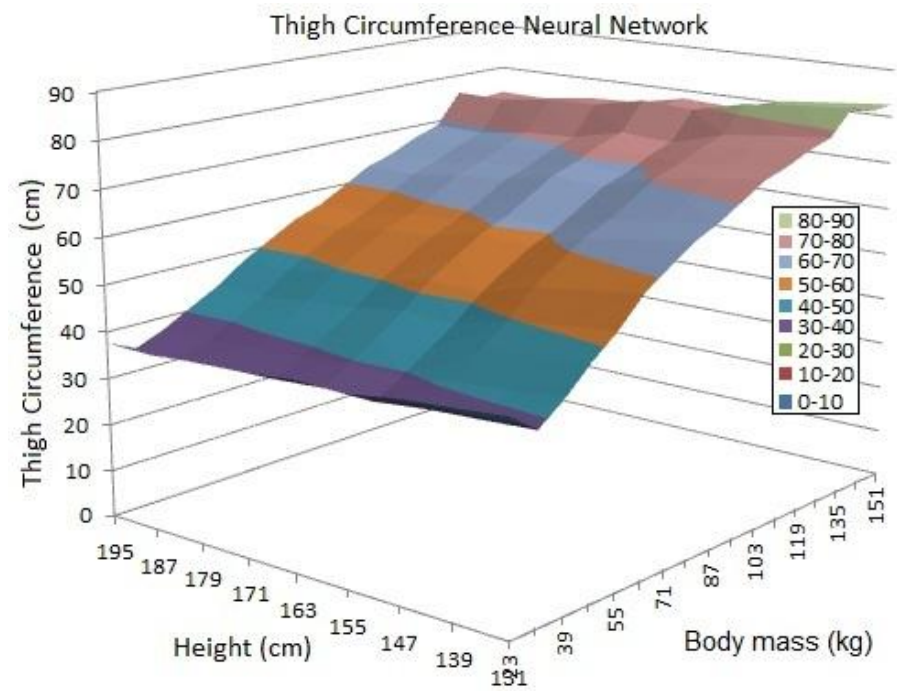

Figure 8. ANN predictions for thigh circumference with mean accuracy within $2.0 \mathrm{~cm}$.

\subsection{Regression analysis results}

The graph in Figure 9 shows the predictions for calf circumference given the intercepts and coefficients from Table 4. This graph for regression analysis provides a comparison to ANN in Figure 6 which shows similar predictions for calf circumference.

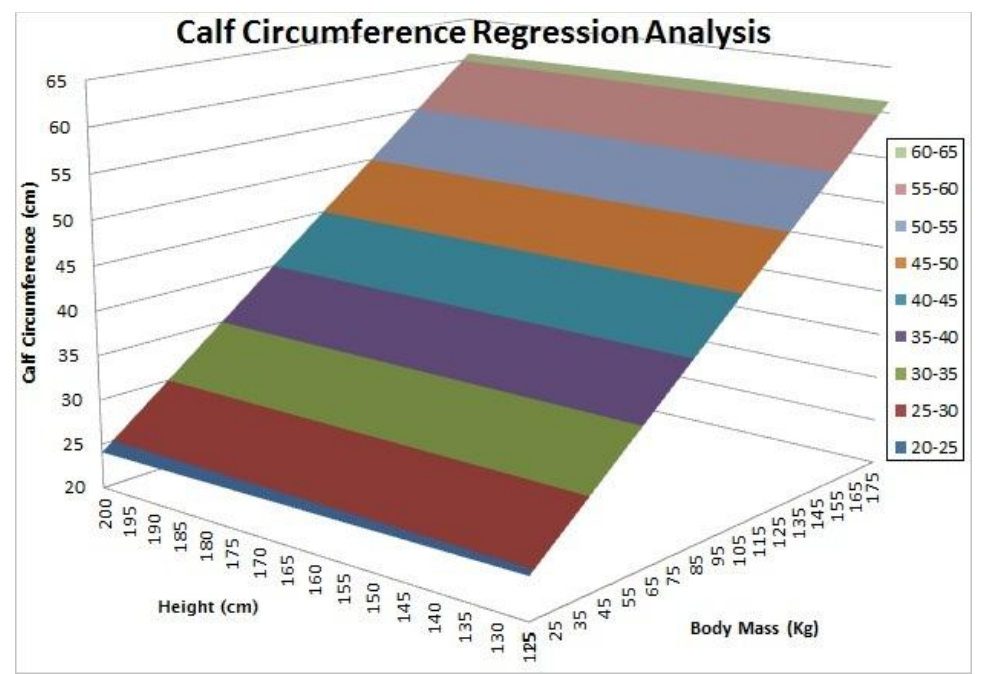

Figure 9. Regression analysis predictions for calf circumference for various patients.

Additional results from regression analysis showed that gender strongly affects skinfold thickness in both triceps and subscapular, see Table 4. This shows that men have on average $3.1 \mathrm{~mm}$ thinner skinfold in subscapular and $7.6 \mathrm{~mm}$ thinner skinfold in triceps thickness which is more than three quarters of a centimeter. This was double checked from the NHANES statistics and found to be correct. This identified gender-based skin thickness change is a new finding which could significantly affect the feeling of epidural procedures between men and women and affects depth to epidural space. 


\subsection{Accuracy comparison of ANN, regression and equations}

The overall accuracy of the fully trained ANN has been assessed in comparison to the body circumference equations (Eqs. 8-12) and regression analysis. An unseen test set containing clinical data from 12088 patients was used. The resulting accuracy comparison is shown in Table 5 .

For all of the estimations, the ANN produced better results than the equations, with up to $54 \%$ less error. BMI was used as an additional assessment parameter which shows that lower errors $(0.69 \%)$ are achievable by the ANN where a strong mathematical relationship exists.

The comparison of regression analysis to the ANN predictions showed close accuracy. Overall, the ANN gave higher accuracy predictions for six of the seven metrics. Regression analysis improved upon ANN accuracy for waist circumference by $0.6 \mathrm{~mm}$. The ANN performed best for arm circumference, showing $1.33 \mathrm{~mm}$ more accuracy than regression.

\begin{tabular}{|l|l|l|l|l|}
\cline { 2 - 5 } \multicolumn{1}{c|}{} & Developed Eq. $(1)$ & ANN $(2)$ & Regression $(3)$ & Difference $(3)-(2)$ \\
\hline Waist & $5.69 \%(7.19 \mathrm{~cm})$ & $3.10 \%(3.92 \mathrm{~cm})$ & $3.036 \%(3.84 \mathrm{~cm})$ & $1.56 \mathrm{~cm}$ \\
\hline Thigh & $4.77 \%(3.39 \mathrm{~cm})$ & $2.81 \%(2.00 \mathrm{~cm})$ & $3.034 \%(2.16 \mathrm{~cm})$ & $1.03 \mathrm{~cm}$ \\
\hline Arm & $3.60 \%(1.75 \mathrm{~cm})$ & $2.48 \%(1.21 \mathrm{~cm})$ & $2.74 \%(1.34 \mathrm{~cm})$ & $0.78 \mathrm{~cm}$ \\
\hline Calf & $4.90 \%(2.01 \mathrm{~cm})$ & $3.40 \%(1.40 \mathrm{~cm})$ & $3.54 \%(1.46 \mathrm{~cm})$ & $0.64 \mathrm{~cm}$ \\
\hline Triceps & $16.35 \%(7.18 \mathrm{~mm})$ & $7.80 \%(3.43 \mathrm{~mm})$ & $8.84 \%(3.89 \mathrm{~mm})$ & $1.62 \mathrm{~mm}$ \\
\hline Subscap & $11.80 \%(4.93 \mathrm{~mm})$ & $8.46 \%(3.54 \mathrm{~mm})$ & $8.96 \%(3.75 \mathrm{~mm})$ & $1.42 \mathrm{~mm}$ \\
\hline BMI & $0.00 \%(0)$ & $0.69 \%(0.46)$ & $0.945 \%(0.63)$ & \multicolumn{1}{|c|}{} \\
\cline { 1 - 5 } & & &
\end{tabular}

Table 5. Error comparison of ANN, regression analysis and equations with clinical data.

When the ANN prediction was too high, often the regression analysis prediction was also too high. This was also true when too low. Therefore the mean difference (Table 5) between the ANN output and the regression output was relatively low, usually less than half of the error of either approach from the actual data. This confirms that the two methods produce similar predictions. Since the two methods are independent this suggests that these results may have reached close to the limit for prediction accuracy based on these polynomials. This could be due to genetics of each patient varying which is not captured in measured data. Also some clinician errors during measurement must play some part including changing position of tape measures between patients, or interoperator variation.

Table 6 shows the standard deviations of prediction values compared to the clinical data. The regression analyses consistently produced lower standard deviation than ANN, 
although on average regression errors were higher than ANN. This may be explained by the regression producing more tightly clustered predictions, as shown in Fig. 10-14.

\begin{tabular}{|l|l|l|l|}
\cline { 2 - 4 } \multicolumn{1}{c|}{} & Developed Eq. & ANN & Regression Analysis \\
\hline Waist & 5.728791 & 3.337018 & 3.101206 \\
\hline Thigh & 2.796388 & 1.911915 & 1.364146 \\
\hline Arm & 1.280478 & 1.123327 & 0.917455 \\
\hline Calf & 1.455131 & 1.255632 & 1.214296 \\
\hline
\end{tabular}

Table 6. Standard deviations of the predicted values from three different methods

Graphs were generated to display a comparison between the actual data, the trained ANN predictions, regression analysis and the equation estimates, shown in Figures 10-14, for calf, waist, arm and thigh circumference and subscapular skinfold for all 23088 patients.

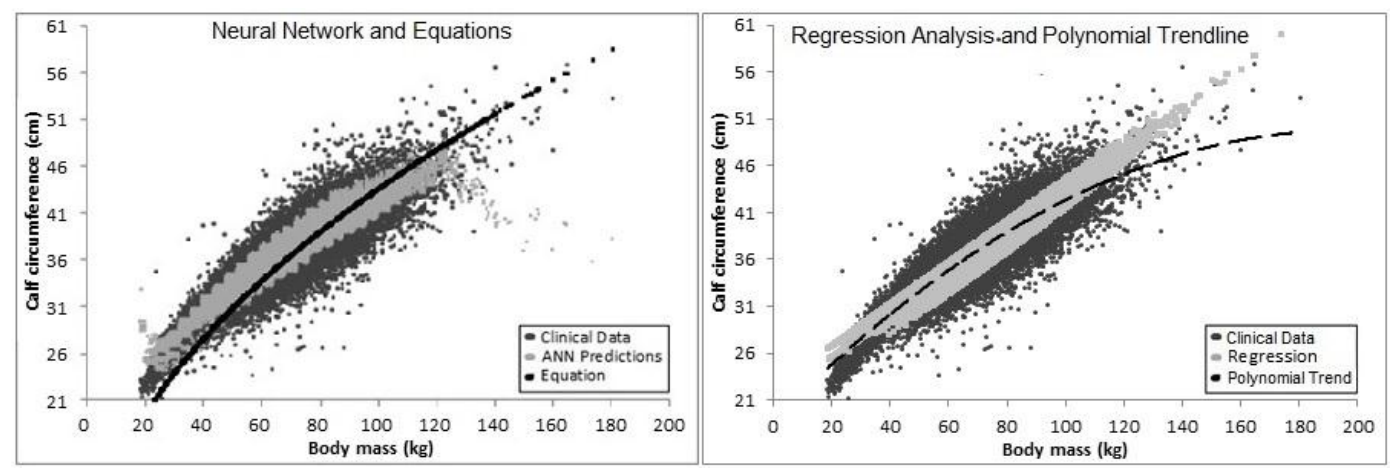

Figure 10. Comparison of calf circumference predictions.
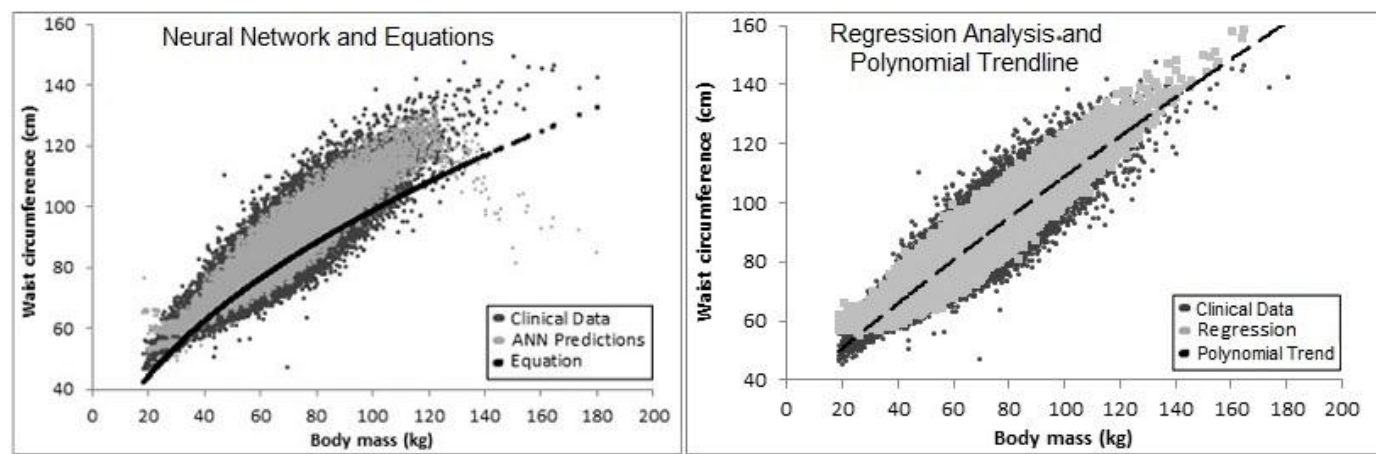

Figure 11. Comparison of waist circumference predictions. 


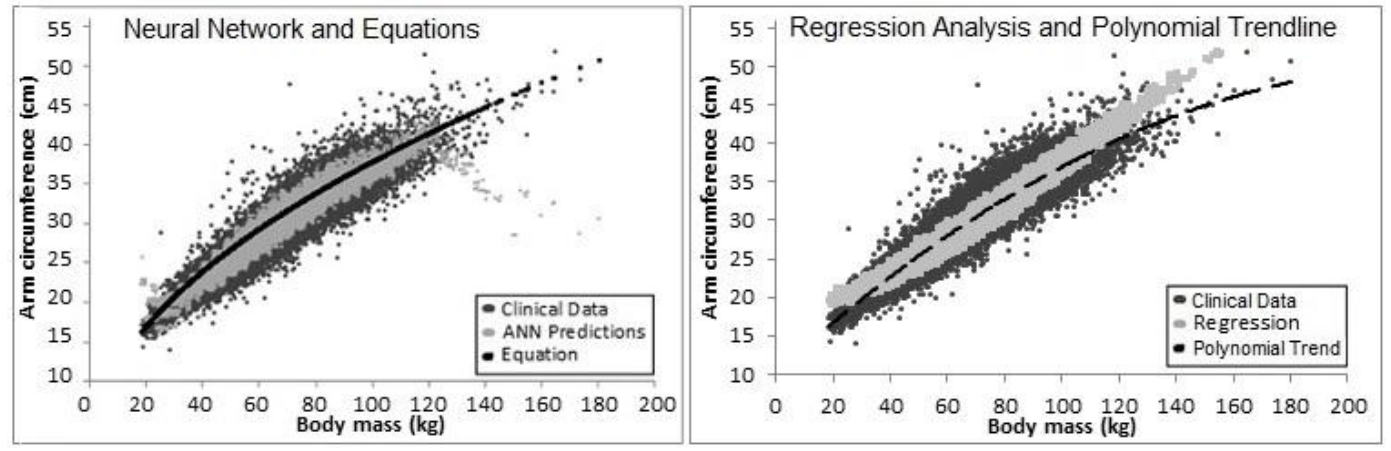

Figure 12. Comparison of arm circumference predictions.
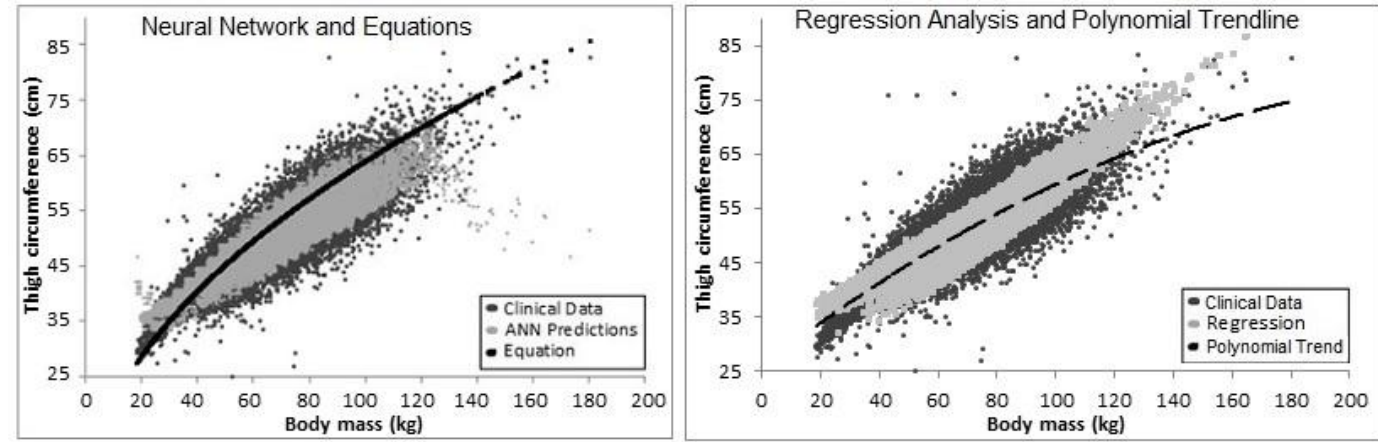

Figure 13. Comparison of thigh circumference predictions.

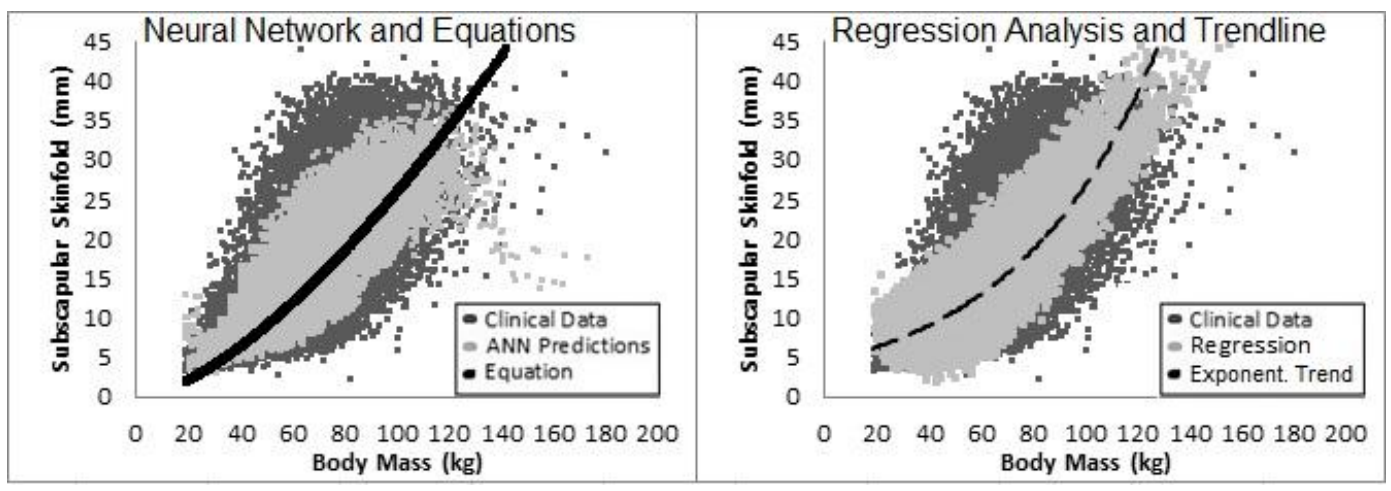

Figure 14. Comparison of subscapular skinfold thickness predictions.

Trend lines are shown on the regression analysis graphs. The best fit were polynomial order two trend lines for the circumferences and exponential trend line for skinfold.

The ANN predictions are clustered close to the clinical data. ANN accuracy was further improved by enlarging training set to 20000 patients and decreasing test set to 3088 . Also the ANN error is reduced if the inputs included decimal places, which they currently do not, although this may increase ANN computation time. One issue that was identified with ANN output was that it is sensitive to gaps in the data. For morbidly obese patients with body mass over 130kg ANN predictions are poor because there were so few such obese patients that the data was insufficient to train the network over $130 \mathrm{~kg}$. This lack of 
data problem also caused the drop in Figure 7 for patients under $131 \mathrm{~cm}$ tall, because there were very few patients in this low height category. However these errors are relatively insignificant because these extreme categories only contain about $0.1 \%$ of total patients. This problem did not affect regression analysis. The equation estimates retain their accuracy even with body masses over 130kg. The equation estimates are linear curves because they are based on body mass but not height. The equation curve is close to the centre of the real data and shows a curve similar to the real data.

\subsection{Body shape visualisation using mesh deformation}

The previous sections have provided methods to describe body shape and ligament thickness, this enables a 3D model to be created to resemble any patient body shape. The body shape model is displayed using a 3D mesh rendered with OpenGL. The challenge of body shape visualization is to ensure that the body shape visualized is a true likeness to a patient of that body shape in vivo. Therefore the methods used to convert body shape data into a 3D model are critical.

Initially a 3D mesh of a female body is used, which was based on average population statistics. The average height and body mass of Japanese 18 to 30 years old are $171.4 \mathrm{~cm}$ and $63.3 \mathrm{~kg}$ [27]. A study of 4243 women in the United States of America [28] suggests that body mass and BMI tend to increase until around the age of 50 and subsequently decrease as shown in Table 7. A separate study matches closely [16] which found women's average body mass to be $67.5-73.6 \mathrm{~kg}$, height $161-168 \mathrm{~cm}$, BMI 25.8 - 28.4. The data in Table 7 was used to generate an average female mesh as shown in Figure 15.

\begin{tabular}{|l|ll|l|l|}
\hline Age & $\begin{array}{l}\text { Body mass } \\
(\mathrm{kg})\end{array}$ & Height $(\mathrm{cm})$ & $\begin{array}{l}\text { BMI } \\
\mathrm{Kg} / \mathrm{m}^{2}\end{array}$ \\
\hline $20-29$ & 71.1 & & 162.8 & 26.8 \\
$30-39$ & 74.1 & & 163.0 & 27.9 \\
$40-49$ & 76.5 & & 163.4 & 28.6 \\
$50-59$ & 76.9 & & 162.3 & 29.2 \\
$60-74$ & 74.9 & & 160.0 & 29.2 \\
$75+$ & 66.6 & & 157.4 & 26.8 \\
\hline
\end{tabular}

Table 7. Mean body mass, height and BMI of American population between 1999-2002 [28] 


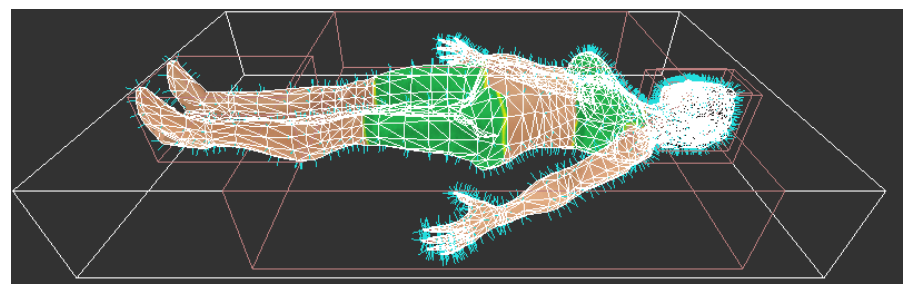

Figure 15. Initial polygon mesh of average female body shape.

An input allows the user to enter basic patient data, the software estimate body size and shape and numerical results are shown on screen (Figure 16). The 3D model is then deformed to match the body shape calculations. Two methods are available to generate results, either the formulae are used to estimate body size and shape and numerical results are shown on screen or alternatively the ANN can be used to generate numerical estimates. The 3D model is then deformed to match the body shape and a rendered 3D model is shown on screen, on which epidural can be performed [29]. The deformation is achieved using the circumference predictions. Algorithms were developed to expand or contract each specific circumference of the body to match predicted values. The algorithms apply vertex buffer objects (VBOs) to quickly deform many vertex positions throughout the model to ensure capability for real-time deformation. Waist deformation is limited to affect solely the waist and surrounding area. Arm circumference is applied to both arms equally primarily on upper arms. Thigh deformation is applied to the area above the knee and below waist.

Alternative methods have been presented in the literature for deforming parameterized human body modelling. Body models have be deformed by inputting a number of sizing parameters based on 3D scanned data, applying both rigid and elastic deformation [30]. Human meshes can be constructed from 3D laser scans and deformed with voxel based parameterization utilizing cloud points, used to model clothing [31]. A body modelling framework was proposed applying whole body scans from 250 subjects and a statistical analysis to correlate several aspects of body shape [32]. Body model shapes can be controlled by parameters learned directly from statistical human body model from 3D scans [33]. Nonlinear optimization has also been applied to extrapolate statistically inferred body shapes to match measurement data [34]. 


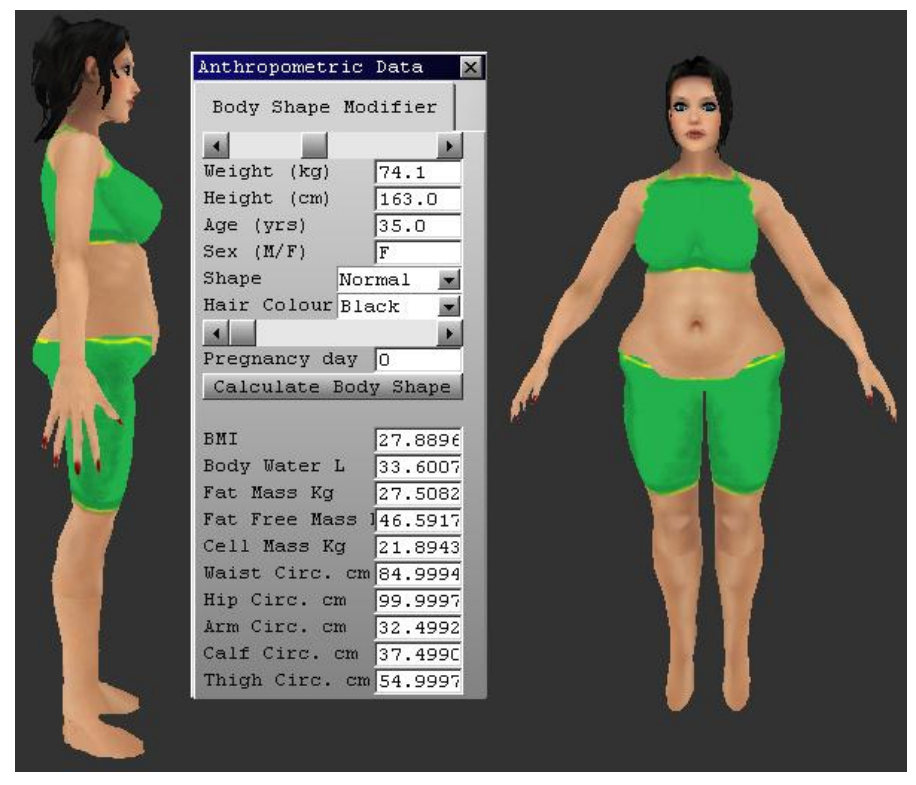

Figure 16. Screen data for entering basic patient data

It is possible to input additional fields if they are known, for example waist circumference. Any extra data overrides the software estimation. The model allows user to input number of days of pregnancy since conception from 0-280. The output from pregnancy model is demonstrated in Figure 17. The whole body experiences growth but the majority is around the bump area.

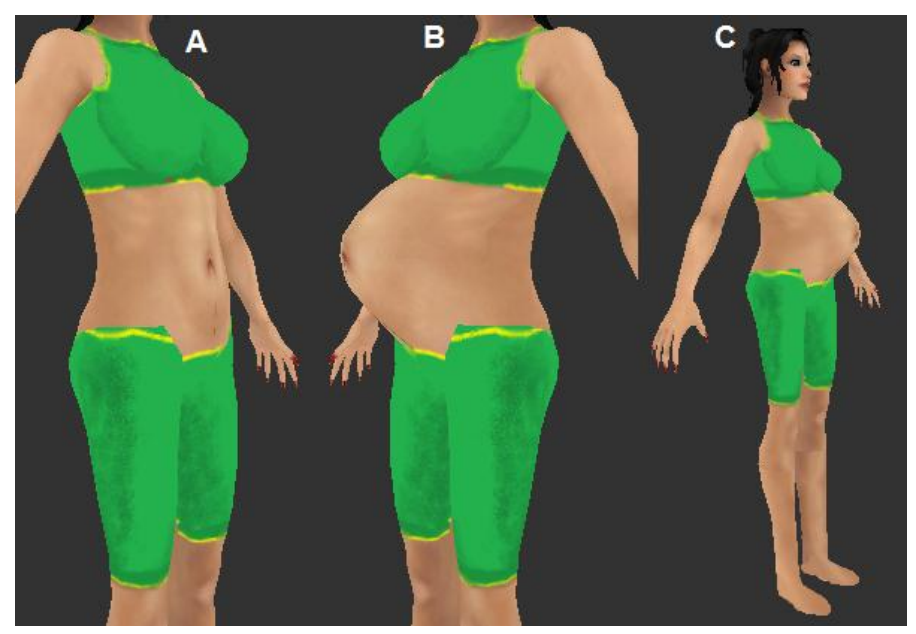

Figure 17. Model of body shape during a) before conception, b \& c) after 40 weeks gestation

\subsection{Needle insertion simulator}

The 3D mesh model was incorporated into an epidural simulator to provide the ability to perform epidural needle insertions on patients of any body mass, height or body shape (Figure 18). The simulator uses the software estimator to visualize a representation of any 
patient from their anthropometric measurements. The model provides depths and thicknesses for the tissue layers and ligaments around the lumbar and thoracic vertebrae which were incorporated into the needle insertion simulation.

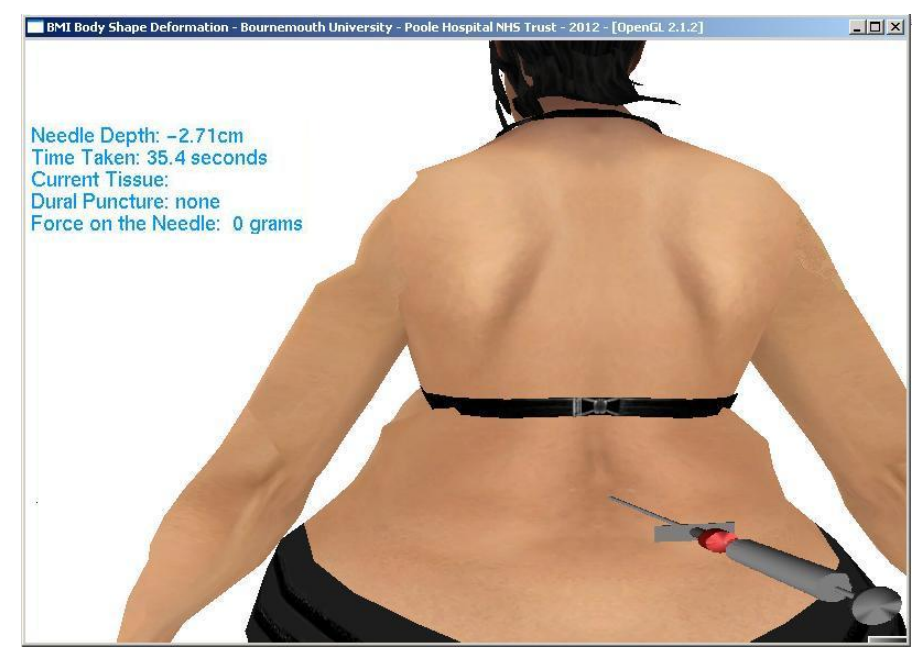

Figure 18. Epidural simulator for patients of any body mass, height or shape.

The developed epidural simulator contains a virtual reality environment allowing the needle to be inserted at various vertebral levels. Haptic force feedback in 3-DOF is based on measurements from the needle insertion trial conducted on a porcine model [29]. The developed simulator provides 3-DOF haptic feedback of needle insertion. Quantifying the adjustment of haptic feedback according to BMI can provide simulation of obese patients which are considered to be difficult. The size and shape of ligaments and vertebrae and the force required for the needle to traverse each tissue layer changes according to patient body shape [25].

\section{Discussions}

In this work three methods for creating a parametric model of human body shape and ligament thickness have been discussed. The first is to use the empirical relationships from equations, the second option is to automatically update the model based on measurements from MRI or ultrasound datasets from the actual patient and the third method is to use ANN to predict body shape and ligament thickness followed by regression analysis on existing data for accuracy comparison. The network gives better accuracy which was trained with 23088 patients. It can further learn by loading data from MRI datasets into a measurement database. The database contains ligament measurements of every patient whose MRI dataset has been loaded into the system. The database is continually growing, so ANN predictions will get more accurate over time. 
The main difficulty of body shape estimation from anthropometrical data is to ensure the visualized body is similar in shape to the real patient. The solution proposed in this work is to develop equations to quantify predictions for body shape, circumferences and body mass gain during pregnancy. The quality criterion for skin and ligament thickness modelling was initially to produce results with higher accuracy. Achieved results from ANN have accuracy within $3.54 \mathrm{~mm}$, sufficient to produce a model of ligament thickness for epidural simulation with regard to training needs and the needs for implementing the approaches for clinical environments. Advantages of the model include visualisation of a general representation of external visual body shape and ability to adjust ligament thicknesses to match specific patients which may be useful for simulating intricate clinical procedures like epidurals.

There are some issues causing complexity to body shape estimation, for example in morbidly obese patients, fat tissue can collect in different parts of the body for each patient. Musculoskeletal proportions may vary between two patients of the same body mass and height. It is not always possible to measure or predict such aspects but this implementation gives a closest approximation based on all known measured data. We are currently conducting a clinical trial which will provide data from MRI and US for the exact thicknesses of ligaments from pregnant women of various BMI categories. The MRI data will allow accurate in-vivo measurement of the depths and thicknesses of the ligaments. This would provide measured data useful for further refinement of the model for estimating body shape and ligament thickness and for simulating various medical procedures.

\section{Glossary}

$\begin{array}{lll}\text { Bioelectrical impedance } & \text { BIA } & \\ \text { Body Adiposity Index } & \text { BAI } & \\ \text { Body Cell Mass } & \text { BCM } & \mathrm{kg} \\ \text { Body Mass Index } & \text { BMI } & \mathrm{kg} / \mathrm{m}^{2} \\ \text { Body Water Volume } & \mathrm{BWV} & \mathrm{Litres} \\ \text { Computed Tomography } & \mathrm{CT} & \\ \text { Epidural Space } & \mathrm{ES} & \mathrm{mm} \\ \text { Fat Free Mass } & \mathrm{FFM} & \mathrm{kg} \\ \text { Fat Mass } & \mathrm{FM} & \mathrm{kg} \\ \text { Height } & \mathrm{H} & \mathrm{cm} \\ \text { Hip circumference } & \mathrm{HC} & \mathrm{cm} \\ \text { Interspinous Ligament } & \text { ISL } & \mathrm{mm}\end{array}$




$\begin{array}{lll}\text { Lean Body Weight } & \text { LBW } & \mathrm{kg} \\ \text { Ligamentum Flavum } & \text { LF } & \mathrm{mm} \\ \text { Magnetic Resonance Imaging } & \text { MRI } & \\ \text { Skeletal Muscle Volume } & \text { SMV } & \mathrm{kg} \\ \text { Subcutaneous Fat } & \text { SF } & \mathrm{mm} \\ \text { Waist Circumference } & \text { WC } & \mathrm{cm} \\ \text { Waist to Hip Ratio } & \text { WHR } & \end{array}$

\section{Conflict of interest statement}

The authors have nothing to declare such as financial and personal relationships with other people or organisations that could inappropriately influence (bias) the work. The work was completed under joint funding from Bournemouth University and Poole Hospital NHS Foundation Trust. The sponsors had a role in design of the study and writing and editing of the manuscript and in the decision to submit the manuscript for publication.

\section{Acknowledgement}

The authors are indebted to joint funding from Bournemouth University and Poole Hospital NHS Foundation Trust during the time of the study.

\section{Ethics}

The dataset does not contain identifying information such as patient images, names, initials, or hospital numbers. The Bournemouth University Ethics service has reviewed the study plan prior to initiation.

\section{References}

[1] O.A. Bamgbade, W.M. Khalaf and A.O. Sharma, Obstetric anaesthesia outcome in obese and non-obese parturients undergoing caesarean delivery: an observational study, Int J Obstet Anesth 18 (2009), 221-225.

[2] D.D Hood and D.M. Dewan, Anesthetic and obstetric outcome in morbidly obese parturients, Anesthesiology 79 (1993), 1210-1218.

[3] J.H. Perlow and M.A. Morgan, Massive maternal obesity and perioperative caesarean morbidity, Am J Obstet Gynecol 170 (1994), 560-565. 
[4] K.N. Elks and R. H. Riley, The Mediseus epidural simulator - does it replicate real patients?, Anaesth Intensive Care 35(5) (2007), 818.

[5] K. Nakamura and T. Kurokawa, An Isomorphic Polygon Model for Describing Human Body Shape, The 6th International Conference on Information Technology and Applications (ICITA), 2009.

[6] P.L. Cornelissen, M.J. Tovee and M. Bateson, Patterns of subcutaneous fat deposition and the relationship between body mass index and waist-to-hip ratio: Implications for models of physical attractiveness, Journal of Theoretical Biology 256 (2009), 343-350.

[7] J. Li, J. Ye, Y. Wang, L. Bai and G. Lu, Fitting 3D garment models onto individual human models, Computers \& Graphics-UK 34(6) (2010), 742-55.

[8] R. Hume, and E. Weyers, Relationship between total body water and surface area in normal and obese subjects. J Clin Pathol 24 (1971), 234-238.

[9] L.S. Piers, M.J. Soares, S.L Frandsen and K. O’Dea, Indirect estimates of body composition are useful for groups but unreliable in individuals, Int J Obes Relat Metab Disord 24(9) (2000), 1145-1152.

[10]P.E. Watson, I.D. Watson and R.D. Batt, Total body volumes for adult males and females estimated from simple anthropometric measurements, Am J Clin Nutr 33(1980), 27-39.

[11] A.R Behnke, Anthropometric Evaluation of Body Composition Throughout Life, Annals of new York academy of sciences 110(2) (1963), 450-464.

[12] A. Sergovich, M. Johnson and T. Wilson, Explorable Three-Dimensional Digital Model of the Female Pelvis, Pelvic Contents, and Perineum for Anatomical Education, Anatomical Sciences Education 3(3) (2010), 127-133.

[13]L.J. Brandon and L. Proctor, Comparison of BMI obesity classification in men and women, Int J Fitness 4(2) (2008), 1-8.

[14]D. Martarelli, B. Martarelli and P. Pompei, Body composition obtained from the body mass index: An Italian study, Eur J Nutr 47 (2008), 409-416.

[15] G.M. Chertow, E.G. Lowrie, N.L. Lew and J.M. Lazarus, Development of a populationspecific regression equation to estimate total body water in hemodialysis patients, Kid Int 51(1997), 1578-1582.

[16] S.B. Heymsfield and A. Martin-Nguyen, Body circumferences: clinical implications emerging from a new geometric model, Nutrition \& Metabolism 5 (2008), 24.

[17] J.E. Brown and E.S.B. Kahn, Maternal nutrition and the outcome of pregnancy: a renaissance in research, Clinics in Perinatology 24(2) (1997), 433-449.

[18]E.P. Gunderson, B. Abrams and S. Selvin, Does the pattern of postpartum weight change differ according to pregravid body size?, International Journal of Obesity 25 (2001), 853862.

[19] N. Vaughan, V.N. Dubey, M.Y.K. Wee and R. Isaacs, Haptic Interface on Measured Data for Epidural Simulation, Proceedings of the ASME Computers and Information in Engineering Conference (IDETC) (2012), 13-17.

[20]E. Zarzur, Anatomic Studies of the Human Lumbar Ligamentum Flavum, Anesth Analg 499(6)3 (1984), 499-502. 
[21] J. Abbas, K. Hamoud, Y.M. Masharawi, H. May, O. Hay, B. Medlej, et al., Ligamentum flavum thickness in normal and stenotic lumbar spines, Spine 35(12) (2010), 1225-1230

[22] T. Sakamaki, K. Sairyo, T. Sakai, T. Tamura, Y. Okada and H. Mikami, Measurements of ligamentum flavum thickening at lumbar spine using MRI, Archives of orthopaedic and trauma surgery 129(10) (2009), 1415-1419.

[23] G. Ayvaz and A.R. Çime, Methods for Body Composition Analysis in Adults, The Open Obesity Journal 3 (2011), 62-69.

[24] J. Wang, J.C. Thornton, S. Kolesnik and R.N. Pierson Jr, Anthropometry in body composition. An overview, Ann N Y Acad Sci 904 (2000), 317-326.

[25] G.A. Bray, K.A. Jablonski, W.Y. Fujimoto, E. Barrett-connor, S. Haffner, R.L. Hanson, et al., Relation of central adiposity and body mass index to the development of diabetes in the Diabetes Prevention Program, Am J Clin Nutr 87 (2008), 1212-1218

[26] Centers for Disease Control and Prevention (CDC). National Center for Health Statistics (NCHS). National Health and Nutrition Examination Survey Data. Hyattsville, MD: U.S. Department of Health and Human Services, Centers for Disease Control and Prevention, 2012 http://www.cdc.gov/nchs/nhanes/nhanes_questionnaires.htm.

[27] T. Nagaoka, S. Watanabe, K. Sakurai, E. Kunieda, S. Watanabe, M. Taki, et al., Development of realistic high-resolution whole-body voxel models of Japanese adult males and females of average height and weight, and application of models to radiofrequency electromagnetic-field dosimetry, Phys. Med. Biol. 49(1) (2004), 1.

[28] C.L. Ogden, C.D. Fryar, M.D. Carroll and K.M. Flegal, Mean Body Weight, Height and Body Mass Index, United States 1960-2002, Advance Data From Vital and Health Statistics 347 (2004), 1-17.

[29] N. Vaughan, V.N. Dubey, M.Y.K. Wee, R. Isaacs, Towards a realistic in-vitro experience of Epidural Tuohy Needle Insertion, Journal of Engineering in Medicine 227(7) (2013), 767-777.

[30] S. Hyewon, and N.M. Thalmann, An automatic modeling of human bodies from sizing parameters, Proceedings of the 2003 symposium on Interactive 3D graphics. ACM, 2003.

[31] C.C. Wang, Parameterization and parametric design of mannequins, Computer-Aided Design, 37(1) (2005), 83-98.

[32] B. Seung-Yeob, and K. Lee, Parametric human body shape modeling framework for human-centered product design, Computer-Aided Design, 44(1) (2012), 56-67.

[33] C. Rupprecht, O. Pauly, C. Theobalt and S. Ilic, 3D Semantic Parameterization for Human Shape Modeling: Application to 3D Animation. IEEE International Conference on 3DTV (2013), 255-262.

[34] S. Wuhrer and C. Shu, Estimating 3D Human Shapes From Measurements, Machine Vision and Applications, 24(6) (2013),1133-1147. 